\title{
Fluoroscopic Management of Complications after Colorectal Stent Placement
}

\author{
Jorge E Lopera* and Miguel Angel De Gregorio ${ }^{\dagger}$ \\ *Department of Radiology, UT Health Science Center at San Antonio, San Antonio, TX, USA, and ${ }^{\dagger}$ Department of Radiology, University \\ Hospital of Zaragoza, Zaragoza, Spain
}

Colorectal self-expanding metal stents have been widely used as a bridge to surgery in patients with acute malignant colonic obstruction by allowing a single-stage operation, or as a definitive palliative procedure in patients with inoperable tumors. Colonic stents are placed under either fluoroscopic or combined endoscopic and fluoroscopic guidance, with similar technical-success and complication rates. Placement of colonic stents is a very safe procedure with a low procedure-related mortality rate, but serious complications can develop and reinterventions are not uncommon. Most of the complications can be treated by minimally invasive or conservative techniques, while surgical interventions are required for most patients with perforation. (Gut Liver 2010;4(Suppl. 1):S9-18)

Key Words: Colorectal cancer; Self-expandable metal stents; Complications

\section{INTRODUCTION}

Colorectal stent placement is now an accepted procedure for the palliative management of colorectal cancer in patients with inoperable disease and as a bridge to surgery in patients with acute colorectal obstruction caused colonic neoplasms involving the rectum and colon. Colonic stenting has technical and clinical success rates of $94 \%$ and $91 \%$, respectively. ${ }^{1}$ Colorectal stent placement can be done using fluoroscopic guidance alone or endoscopic and fluoroscopic guidance. Technical results are similar between the two techniques in the majority of series. ${ }^{2}$ Endoscopic guidance is very useful for proximal lesions and when very tortuous anatomy is present. In re- cent years dedicated colonic stents have been available with more flexible stents and longer delivery systems that have resulted in lesser incidence of device related complications, despite these recent advances the overall reintervention rate is still high and the ideal colorectal stent is yet to be developed. ${ }^{3}$ Complications after colorectal stent placement are usually minor but severe life-threatening complications can developed any time after the procedure. The purpose of this article is to review the management of complications after colorectal stent placement from an interventional radiologist's perspective.

\section{COMPLICATIONS AFTER COLORECTAL STENT PLACEMENT}

Complications can be divided in early ( $<30$ days) and late (>30 days) complications. Complications are also classified as minor complications when they are self-limited and not requiring additional intervention; and major complications when additional radiological, endoscopic or surgical interventions are required, hospital admission is needed or the complication lead to patient's death. Table 1 lists incidence of major and minor complications.

\section{Factors associated with complications}

Although some complications are unavoidable, certain factors can be associated with a higher incidence of major complications.

\section{1) Operator experience}

Excessive manipulation can be associated with perforation, most of the time caused by the guidewires trying to cross the obstruction (Fig. 1). Also inadequate center-

\footnotetext{
Correspondence to: Jorge E Lopera

Department of Radiology, UT Health Science Center at San Antonio, 7703 Floyd Curl Drive, San Antonio, TX 78229, USA

Tel: +1-210-567-5564, Fax: +1-210-567-1494, E-mail: Lopera@uthscsa.edu
}

DOI: $10.5009 /$ gnl.2010.4.S1.S9 
Table 1. Minor and Major Complications after Colonic Stent Placement

\begin{tabular}{lclc}
\hline Minor complications & Incidence, $\%$ & Major complications & Incidence range (mean), $\%$ \\
\hline Bleeding & $8-12$ & Migration & $8-36(10)$ \\
& & Uncovered stents & $8-50$ \\
Pain & 5 & Covered stents & $2-10(4)$ \\
Tenesmus & 5 & Perforation & $3-62(7)$ \\
Incontinence & 11 & Reobstruction: & \\
Fecal impaction & 25 & Tumor overgrowth & Rare \\
Bacteremia/fevers & 3 & Tumor ingrowth & $0-15(0.5)$ \\
& & Stent related collapse & Death related to complications \\
\hline
\end{tabular}
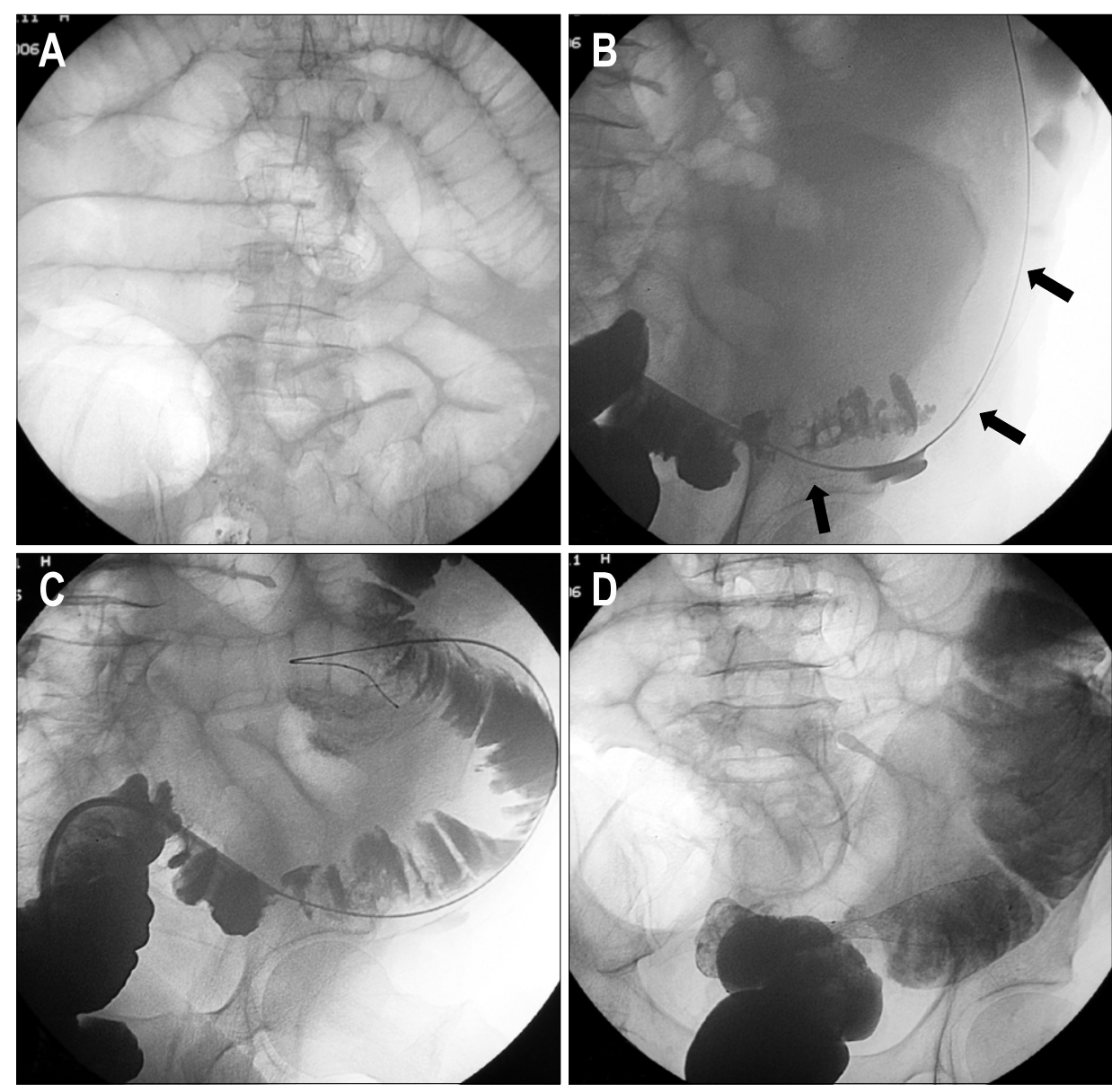

Fig. 1. A 64-year-old female with acute left colonic obstruction from a rectosigmoid cancer. (A) AP plain film of the abdomen shows severe dilatation of the intestinal loops. (B) Spot radiograph obtained during fluoroscopyguided stent placement shows the guide wire outside the colonic lumen (arrow) as a result of iatrogenic perforation. (C) Spot radiograph shows that the position of the guide wire was corrected to the intraluminal position. (D) Radiograph after Wallstent deployment showing the stent in an adequate position. The acute obstruction resolved with no signs of peritonitis, and the stent was used for long-term palliation.

ing of the stent in the lesion can result in early stent migration. Placing a stent that is too short with inadequate covering of the tumor margins can be associated with lack of resolution of the obstructing symptoms. Several studies have shown that complications are more common when the procedure is performed by less experienced operators. ${ }^{1,4}$

\section{2) Type of stent}

Certain stents maybe too rigid for the normal curva- tures of the colon. A study by Small and Baron, ${ }^{5}$ demonstrated that the use of the Wallstent (Boston Scientific, Natick, MA, USA) was associated with higher incidence of technical difficulties such as insufficient stent expansion and stent misplacement Fig. 2; higher incidence of major complications such as perforation, stent occlusion, migration, stent erosion/ulcer and stent collapse than the Ultraflex stent (Boston Scientific). The overall reintervention rate was $62 \%(31 / 50)$ for the Wallstent group and $40 \%$ for the Ultraflex group (14/35). Some of 

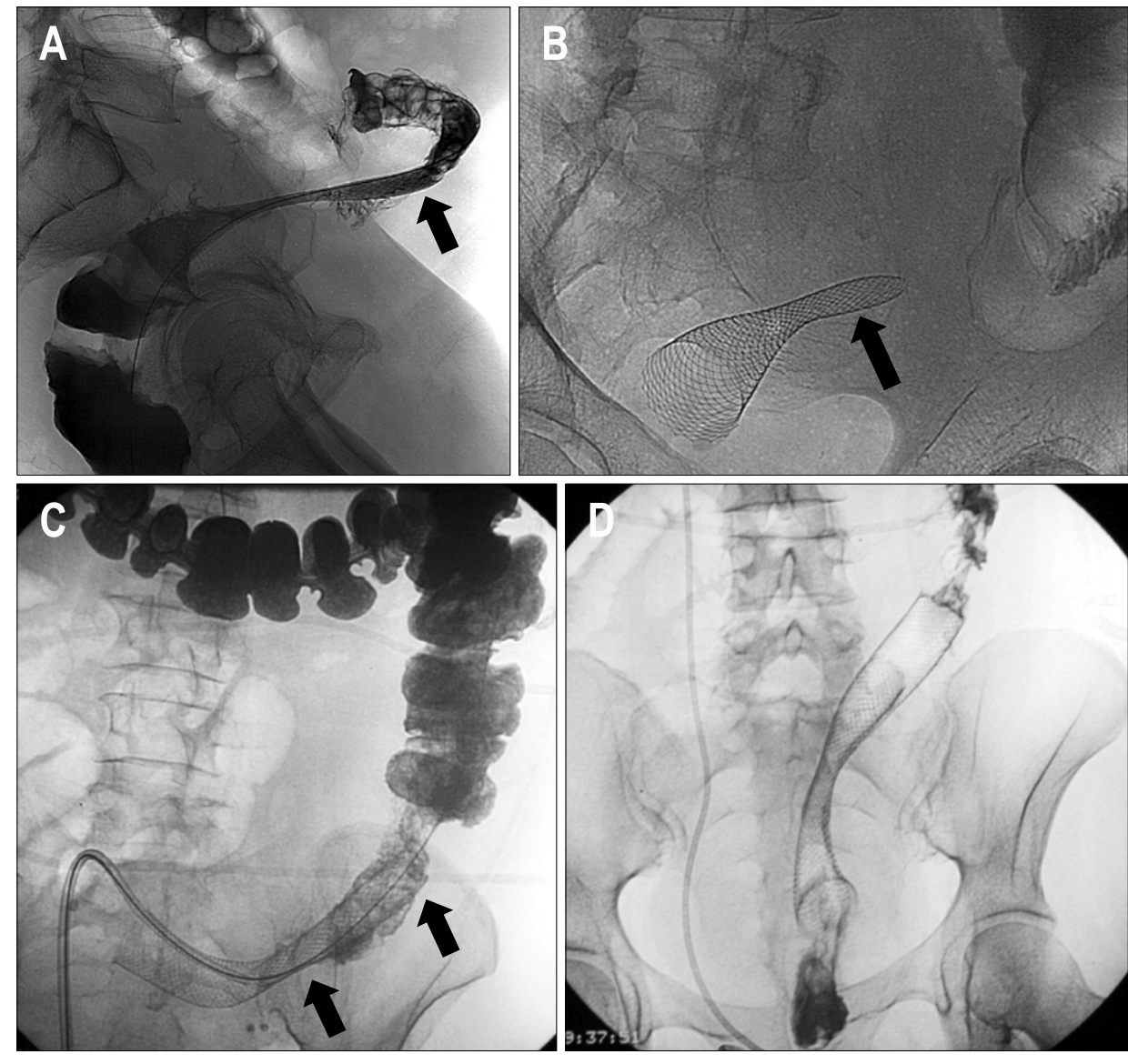

Fig. 2. A 55-year-old female with an obstructing sigmoid carcinoma. (A) Lateral radiograph after Wallstent deployment shows inadequate expansion of the proximal end of the stent (arrow). The design of the Wallstent is associated with a higher incidence of inadequate stent expansion either when the stent is too short or when it is not adequately centered in the obstruction. (B) Plain radiograph obtained 24 hours after the procedure shows persistent collapse of the proximal end of the Wallstent, with persisting intestinal obstruction. (C) Reintervention involved the placement of an overlapping Wallstent. Radiograph shows the second stent placed proximally (arrows). (D) AP radiograph obtained 24 hours after the reintervention shows adequate expansion of the two stents with resolution of the intestinal obstruction. Note the interval placement of a double-J right ureteral stent. these complications can be explained by the smaller caliber of the Wallstent $(<22 \mathrm{~mm})$, than that of the Ultraflex (25 $\mathrm{mm}$ in the main body, $30 \mathrm{~mm}$ flared ends). Perforation can also be associated with the delivery system of the stent, Song et al., reported a high incidence of perforation with the use of dual colorectal stent due to the rigidity of the delivery system. ${ }^{6}$ Covered stents have been used to prevent reocclusion of the stent by tumor ingrowth but are associated with a much higher incidence of stent migration. ${ }^{7}$

\section{3) Type of stricture}

(1) In many series, extrinsic lesions are associated with less clinical success, more complications and higher need for surgical diversion. ${ }^{1,8,9}$ Extrinsic compression causing colonic obstruction is frequently associated with peritoneal carcinomatosis with bowel immobilization and higher incidence of prior radiation. Higher incidence of stent migration, perforation, need for surgery and procedure related mortality has been reported in patients with extrinsic compression of the rectosigmoid area after placement of colonic stents. ${ }^{8,9}$ Patients with extrinsic obstructions also have a higher incidence of additional lesions in other parts of the colon and small bowel.
However, a study by Shin et al. ${ }^{10}$, reported a success rate of $82 \%$ in patients with extrinsic compression with a similar rate of complications than patients with intrinsic lesions, most of the obstructions in this study were located in the transverse after bowel invasion from advanced gastric cancer.

(2) Longer strictures $(>10 \mathrm{~cm})$ also had worse outcomes than shorter strictures ${ }^{11}$ possibly due to longer strictures tend to involve the curvatures of the colon. Angulated lesions have higher rate of stent migration and perforations, these type of lesions are usually seen in the rectosigmoid area.

(3) Lesions of the right and proximal colon are much difficult to treat by fluoroscopic methods alone and endoscopic assistance is many times required. Placement of stents in the proximal colon using cecostomy access is an alternative when other methods have failed (Fig. 3). Many series report similar or better clinical results in lesions of the proximal colon. ${ }^{4,12}$

(4) Degree of obstruction: Some series report a higher incidence of overall complications when the obstruction is complete, ${ }^{4}$ this could be related to microperforations caused by the complete obstruction and/or technical difficulties when crossing the lesions with higher manipu- 

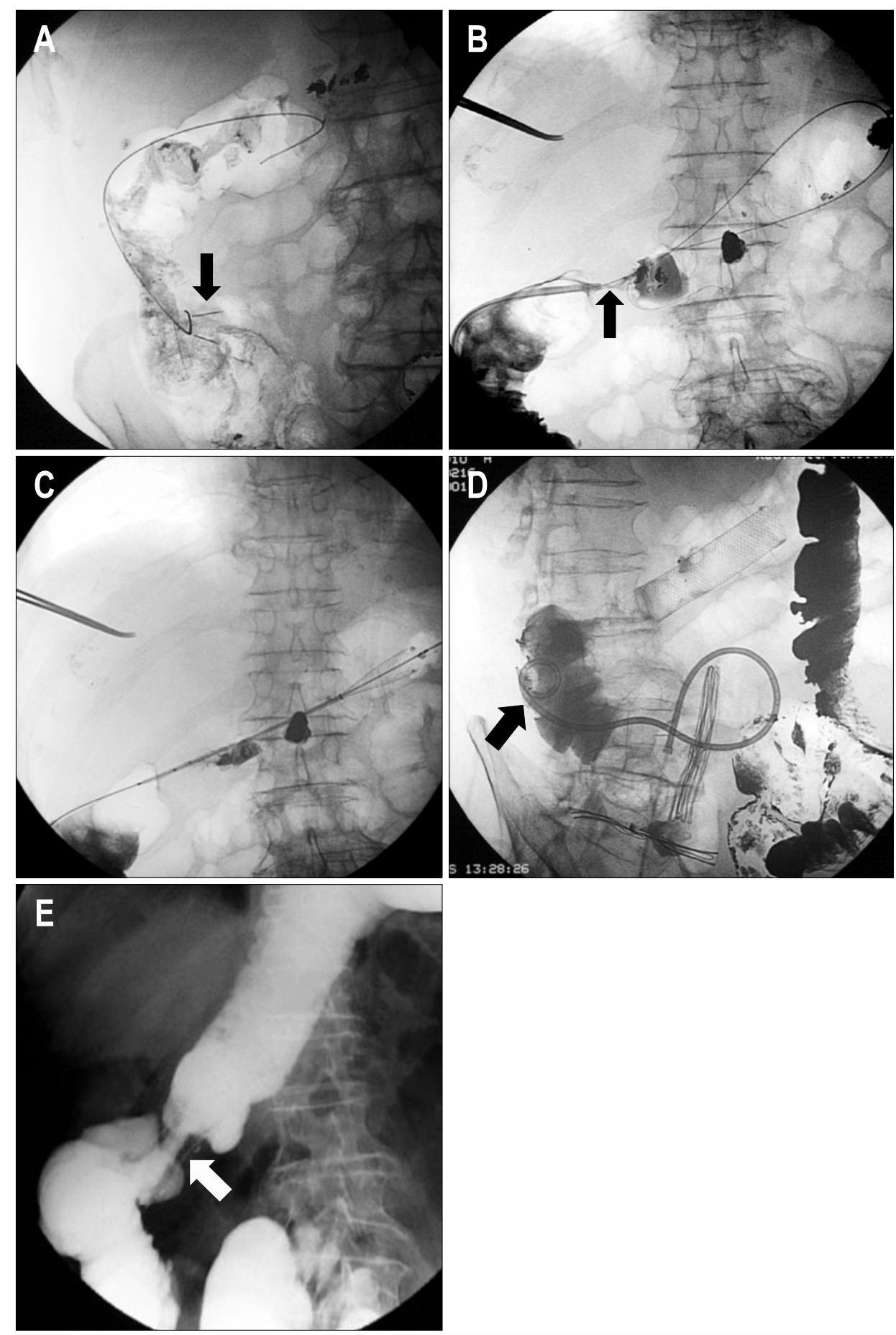

Fig. 3. A 72-year-old male with obstructing colon carcinoma of the hepatic flexure. Severe tortuosity of the left colon prevented stent deployment using a transanal approach. (A) Radiograph shows cecostomy access created after deployment of two T-fasteners (arrows). (B) Hydrosoluble contrast-agent enema shows that the proximal lesion (arrow) was spanned by the guide wire. (C) Radiograph shows deployment of the Wallstent. (D) Oblique projection of the hydrosoluble contrast-agent enema showing adequate expansion of the stent. Note that the pigtail catheter (arrow) was left in place until the cecostomy access tract healed. (E) Control barium enema showing the contrast agent passing adequately through the stented area (arrow). 
lations. Others reported higher clinical failures in case of complete obstructions. ${ }^{13}$

\section{4) Use of chemoradiation}

The use of chemoradiation before and after colonic stents has been associated with a higher incidence of perforation and stent migration. ${ }^{14,15}$ Migration can be explained by improvement in the stenosis with tumor shrinkage after chemoradiation. In many of these patients there is no recurrence of the obstruction after stent migration and additional interventions may not be required. The recent use of Bevacizumab (Avastin Genentech Inc., San Francisco, CA, USA), an antiangiogenic agent in combination with other chemotherapy drugs have resulted in higher bowel perforation rates compared with controls in the absence of colonic stent placement. ${ }^{4}$ Perforations are more common and also appear to occur earlier in patients with palliative colonic stents taking Bevacizumab. ${ }^{4}$

\section{Complications and its management}

\section{1) Minor complications}

(1) Bleeding: The most common complication in this category, bleeding is usually related to the pressure of the stent against friable tumor. In the majority of the cases the hematochizia is resolved with conservative treatment, ${ }^{12}$ blood transfusion and surgical intervention are rarely required. Late bleeding can be related to erosion/ulcers of the colonic mucosa by the stents. Occasionally radiation colitis may cause bleeding after stent placement.

(2) Pain and tenesmus: Pain is one of the most com- mon complains after stent placement. It is usually self-limited and tends to improve with time. If post-stenting pain becomes severe, care should be taken to rule out serious complications such as perforation and/or stent migration. Migration of stents into the anorectal area is also a potential source of significant pain that does not respond to analgesics. ${ }^{16}$ In a retrospective study, Song et al. ${ }^{16}$ studied the tolerance of rectal stents placed within 5 $\mathrm{cm}$ of the anal verge, 10 of 16 patients with obstruction within $5 \mathrm{~cm}$ of the anal verge complained of severe pain with foreign body sensation when sitting upright, with 7 of these patients requiring narcotics for analgesia until death or surgery, while only 1 of 14 patients with obstruction beyond $5 \mathrm{~cm}$ had this problem.

Retrievable stents seem to be a good choice for patients with low rectal obstructions, since pain, incontinence, or tenesmus are potential severe problems that could be not reversible unless the stents are removed. ${ }^{3}$ Currently several retrievable colonic stents are available in Europe and Asia Fig. 4, but none in the USA.

(3) Fecal impactation: Fecal impaction usually presents as sudden onset of bowel obstruction. Diet counseling with a high fiber diet and routine use of laxatives are measures that may help prevent impaction. Once impaction is suspected, cleaning enemas are performed. Impaction may require the radiologist to perform a water soluble enema to diagnose the obstruction followed by flushing of the colon with saline. Endoscopic lavage is also frequently performed. In some cases lavage is not sufficient and mechanical recanalization under fluoroscopic or endoscopic guidance may be required to relieve the blockage. When intervention is required to relieve the im-
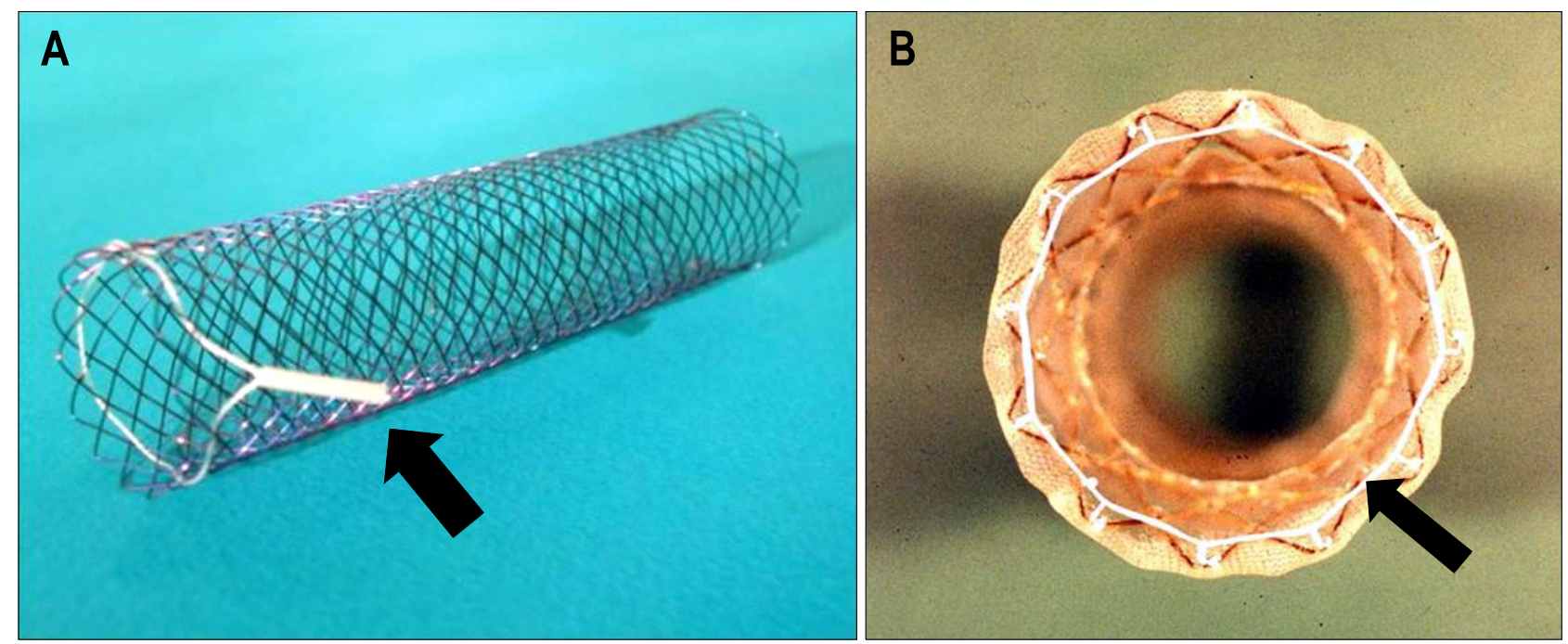

Fig. 4. Photographs of two colonic stents with retrieval systems consisting of a drawstring suture (arrow). (A) Ella stent (CS-ELLA, Czech Republic). (B) Song's colonic stent. 

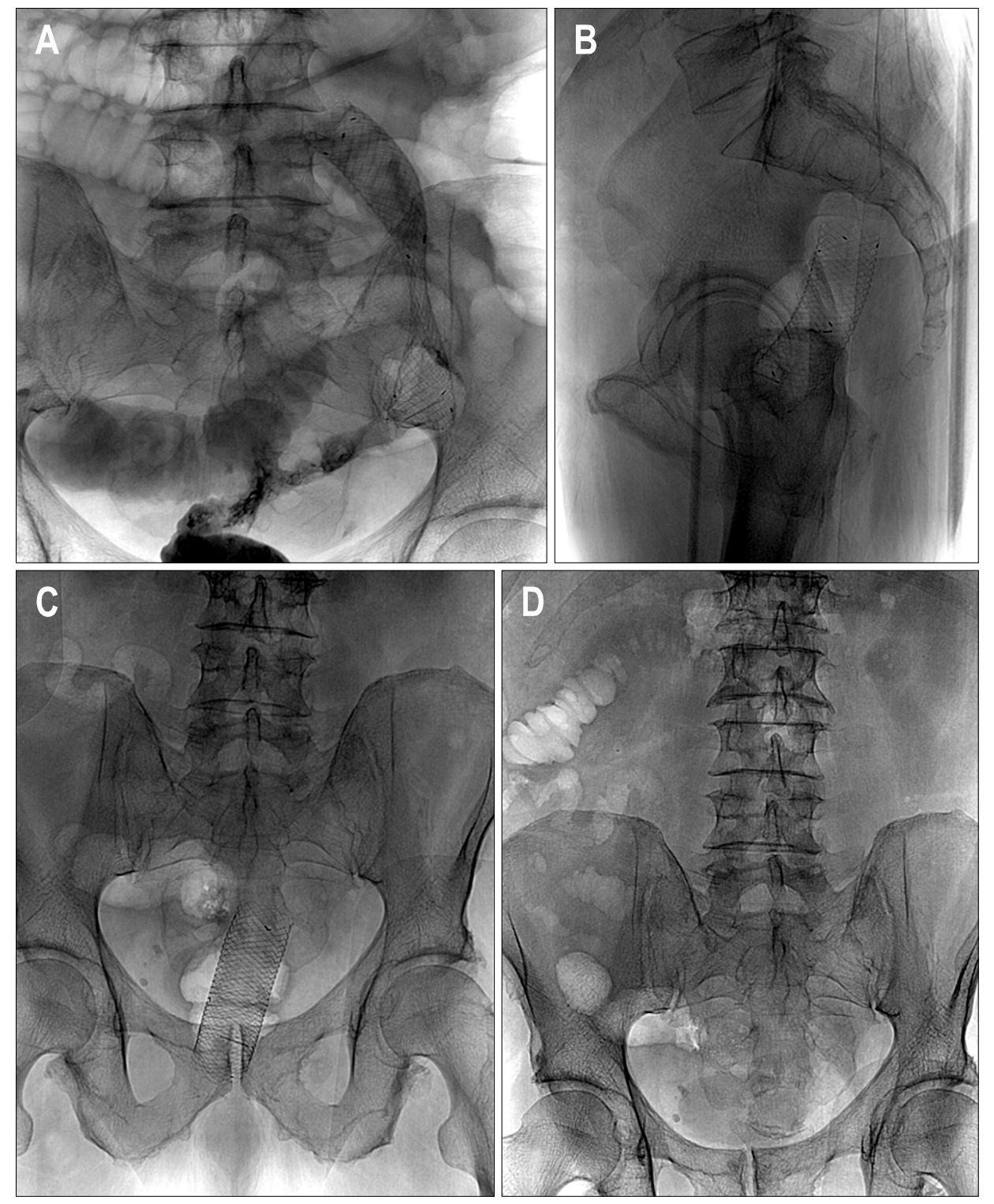

Fig. 5. A 61-year-old female with obstructing carcinoma of the sigmoid colon. (A) AP radiograph shows a Wallflex stent (Boston Scientific) successfully placed for palliation in the rectosigmoid colon. (B) Lateral and AP (C) radiographs taken 6 days later show migration of the stent into the rectum. (D) Radiograph taken 9 days after stent placement shows absence of the migrated stent due to its spontaneous passage. The bowel obstruction was relieved and the patient underwent elective surgical resection 1 week later.
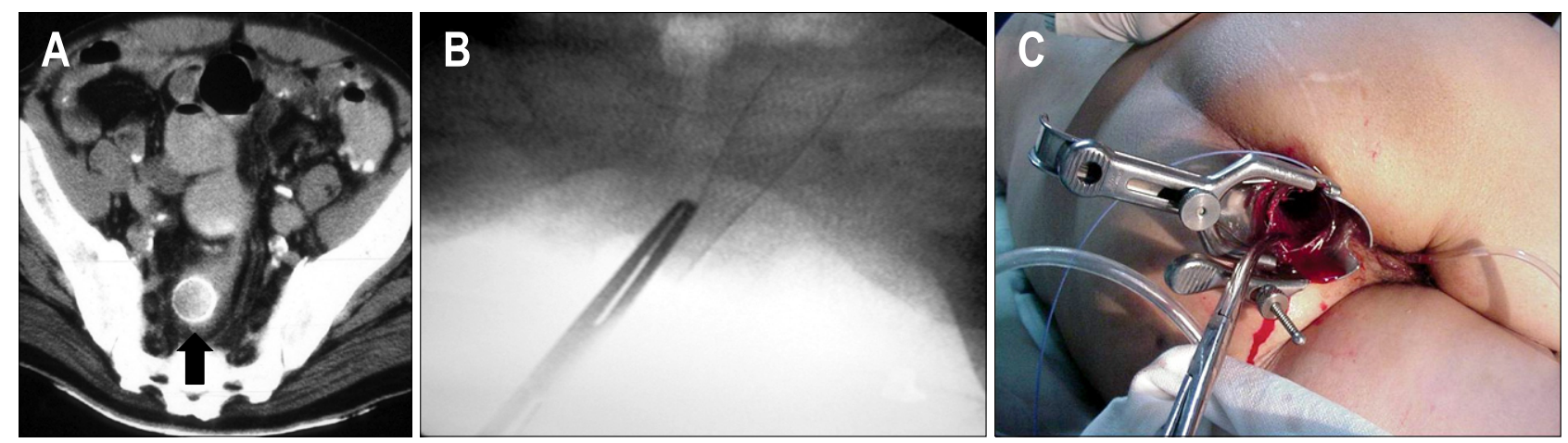

Fig. 6. (A) Axial CT scan shows a migrated Wallstent (arrow) that had been placed in the rectosigmoid colon 24 hours earlier. (B) Radiograph shows the use of forceps under fluoroscopic guidance to grab and collapse the stent. (C) Photograph shows the use of a speculum to protect the anal mucosa when forceps are used to remove a migrated Wallstent. 
paction, this complication is classified as a major complication.

(4) Incontinence: This could be a very disabling complication that usually occurs after placement of low rectal stents that may interfere with the anal sphincter function. This complication may require removal of the stent. Patients with very low tumors need to be counseled about this possible problem before the procedure is performed.

(5) Bacteremia and fever: This is an infrequently reported complication, transient bacteremia produced by enteric bacteria has been reported ${ }^{4}$ and appears more frequently is cases of total obstruction, for this reason many authors recommend peri-procedural broad spectrum antibiotics.

\section{2) Major complications}

(1) Migration: Migration rates associated with uncovered stents have ranged from $3 \%$ to $12 \%$. The overall migration rates for covered stents are reported to be as high as $30 \%$ to $50 \% .^{1,17,18}$ Migration can occur early and can be related to initial malposition of the stent. To avoid migration it is important to center the stent in the stricture and always use a longer stent that covers $2-4 \mathrm{~cm}$ of normal colon proximal and distal to the lesion. Manipulation of the stent by rectal exams and colonoscopy can cause displacement of the stents. Migration is

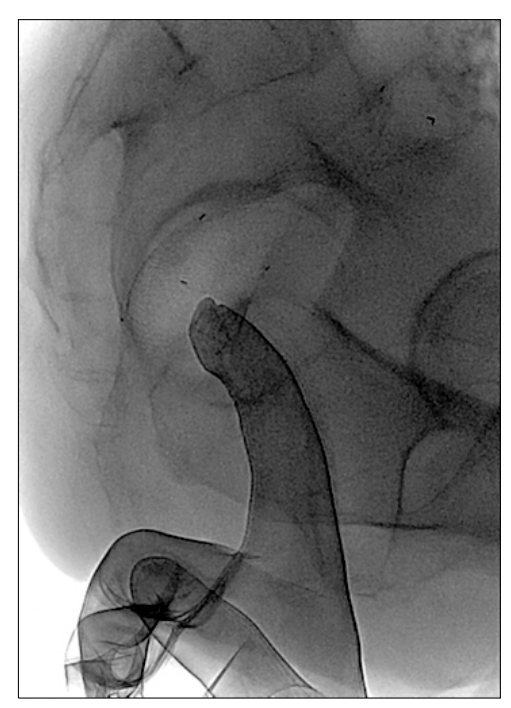

Fig. 7. Lateral radiograph shows the finger technique used to remove migrated stents. Under fluoroscopic guidance and using radiation-reducing gloves, the stent is carefully collapsed and retrieved using one finger. This technique is not recommended when removing a Wallstent since there is a high risk of the sharp ends of the stent accidentally injuring the fingers of the operator. more frequent after chemotherapy, laser pretreatment, and dilatation prior to stent insertion, as well as in patients with strictures of benign etiology. ${ }^{1}$ Migration can lead to recurrence of the obstructive symptoms, but in many cases the improvement of the stricture after chemoradiation leads to the migration and additional intervention may be not required. ${ }^{19}$ In most instances, the stent migrates distally and sometimes the stent passes out through the anus (Fig. 5). However, the moving distal end of the migrated stent can cause pain or even perforation by the continuous irritation of the colorectal wall. $^{2}$ Migration of the stent proximal to the stricture is also possible, these stents are usually not removed due to the technical difficulties, restenting the obstruction is usually performed and the migrated stent is left in place.

Fluoroscopic techniques to remove migrated colonic stents: Retrieving stents is usually a very uncomfortable and painful procedure for patients especially when significant manipulation is required. Care should be taken to avoid perforation or injury of the rectal mucosa during stent retrieval. The first step is to identify the type of migrated stents, rigid stents such as the Gianturco $\mathrm{Z}$ stent (Cook, Bloomington, IN, USA) are more difficult to remove. The Enteral Wallstent has multiple sharp edges and retrieval could cause significant injury to the rectum. The use of a plastic sheath through anus as protection such as a gynecologic speculum has been reported Fig. 6. ${ }^{20}$

Migrated stents can be retrieved using a variety of techniques. The choices for fluoroscopic retrieval are more limited that the endoscopic techniques. Reported techniques include:

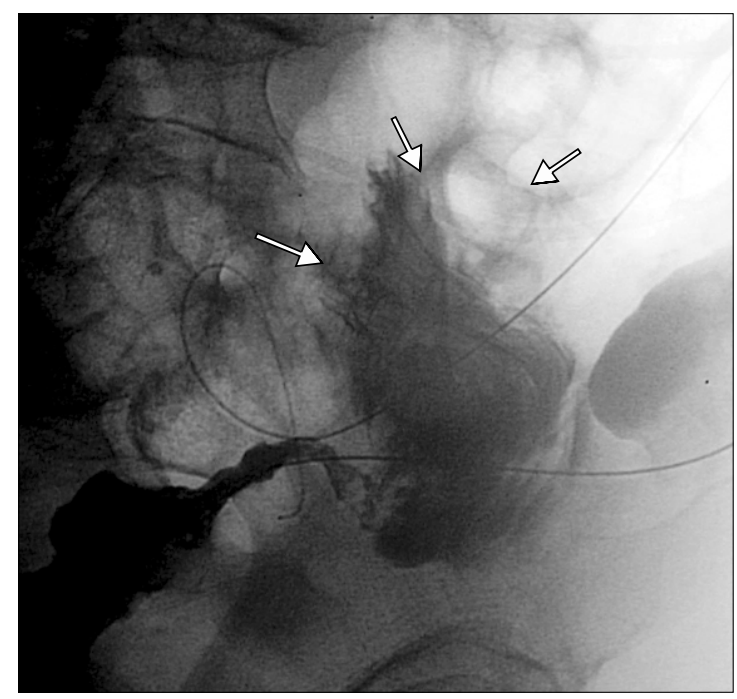

Fig. 8. Hydrosoluble contrast-agent enema during stent deployment shows frank perforation with extravasation of the contrast agent (arrows). Emergency surgery was performed. 

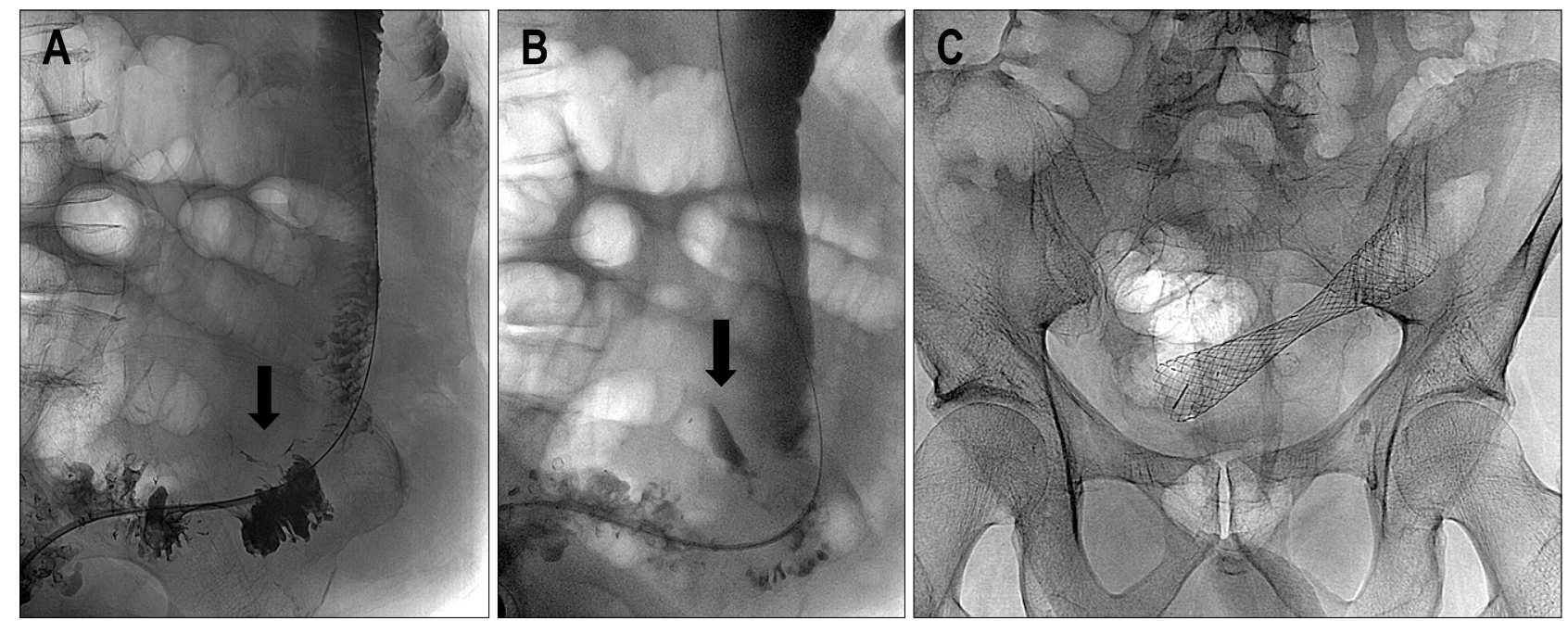

Fig. 9. (A) Hydrosoluble contrast-agent enema during stent deployment shows limited perforation (arrow). (B) A delayed film shows focalized collection of the contrast agent (arrow). (C) The stent was deployed and the patient was carefully observed for signs of peritonitis. Radiograph showing the stent in an adequate position. The patient underwent elective surgery 12 days later, during which a limited area of inflammation was noted around the perforation.

Snare technique: The proximal end of the stent closer to the rectum is collapsed after snaring it, and then the stent is retrieved. This maneuver may be possible in the rectum but many times the ends of the stent are opposed against the wall and it is not possible to pass the snare over the stent. Snaring of wire passed through the stent lumen may allow folding the stent and then retrieving it in a folded position.

Forceps removal: Under fluoroscopic guidance a forceps is use to grasp and collapse a low lying stent (Fig. 6).

Finger extraction of the stent is also possible, with the operator wearing a radiation attenuating glove, a finger is advanced inside the stent and the stent is removed partially either partially folded or partially collapsed by the finger (Fig. 7).

Retrievable stents: Many colorectal stents used in Asia and Europe have a retrieval systems that allow easy and atraumatic removal. The drawstring of the systems allows collapsing the distal end of the stent to facilitate retrieval. ${ }^{21}$ The drawstring can be grabbed with a special hook under fluoroscopic guidance or with a forceps under endoscopic guidance. In some cases the drawstring is broken or embedded in the mucosa and may not be reachable, stent retrieval can be performed using the eversion technique where the more distal end of the stent is grabbed with the hook and the stent is everted and retrieved. ${ }^{21}$ Another described technique is to grab the proximal mesh at the end of the stent and retrieve the stent in an expanded form. ${ }^{21}$ Some stents can become encased by the colonic mucosa and may be impossible to remove; excessive manipulation may result in mucosal in-

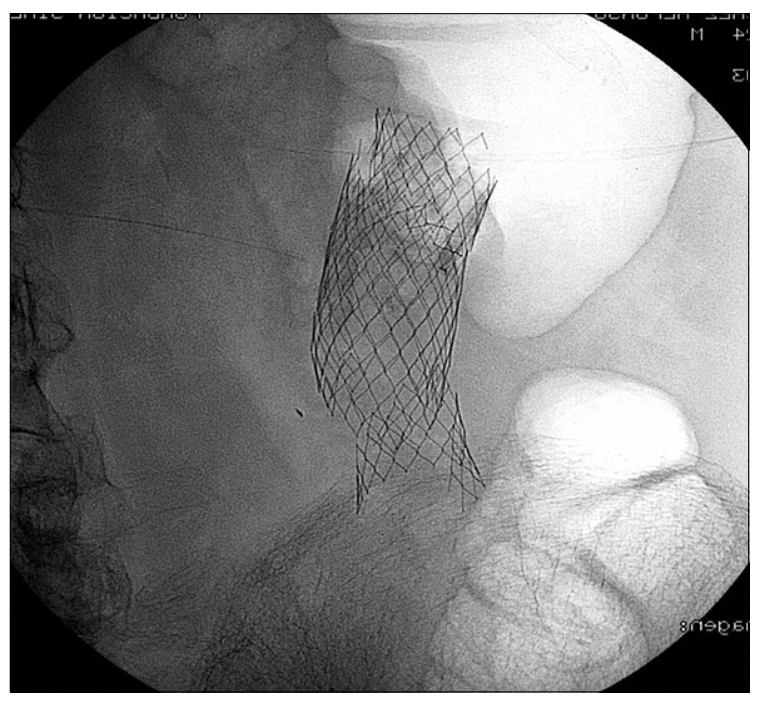

Fig. 10. A 65-year-old male underwent palliative placement of a Memotherm (Bard, Billerica, MA, USA) colonic stent. The patient presented with signs of intestinal obstruction at 6 months after placement. Radiograph shows that the stent had fractured at several locations. The patient was treated surgically.

jury and perforation or severe bleeding. ${ }^{16}$

(2) Perforation: Perforation is the most serious complications after colorectal stent placement and the leading cause of death related to the procedure. Many patients with malignant colonic obstruction have advanced disease and are in a poor medical condition. These patients may not tolerate an exploratory laparotomy and may die soon after the perforation with or without surgery. ${ }^{15}$ Perfora- 

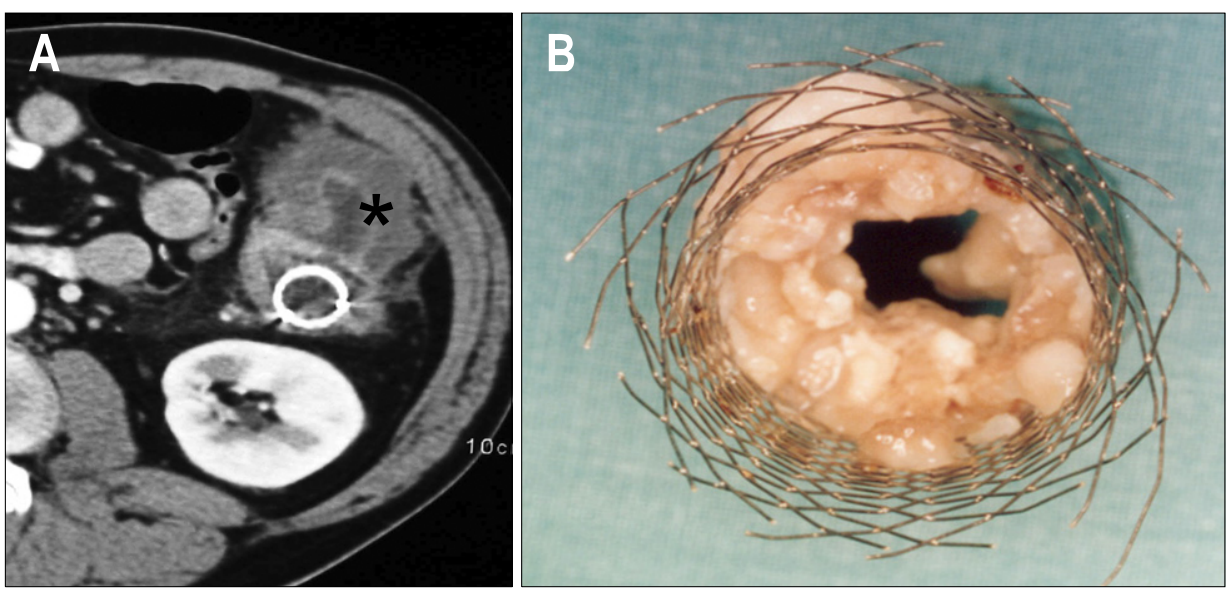

Fig. 11. A metallic stent had been placed in the descending colon of a 54-year-old patient 6 months earlier. (A) Computed tomography axial image shows a large tumoral mass (asterisk) in the descending colon with occlusion of the stent lumen. (B) Surgical specimen shows the Wallstent with occlusion of the lumen due to tumor ingrowth.

tion should be suspected when patients develop symptoms of peritonitis (severe pain, fever, leukocytosis) or when free gas is detected at erect radiography after stent placement. Minimal amounts of free intraabdominal gas are better detected with computed tomography. Perforation can be confirmed as extraluminal contrast leakage on water soluble contrast enemas (Fig. 8). Perforation usually occurs in the first 3 days after stent placement. The incidence varies between $5-16 \%$. The greatest risk of perforation is in the rectosigmoid area. Perforation is usually an acute complication and procedure related. Excessive manipulation with the guidewire, more commonly in higher degrees of obstructions and procedures performed by inexperienced operators, are potential causes of acute perforation. Balloon dilation before stent placement has been associated with a higher incidence of perforation and its routine use is not recommended. Perforation can also be related to unsuccessful bowel decompression after the procedure. ${ }^{22}$ Late perforation is related to stent pressure into the tumoral area and it is usually stent related. Perforations are also caused by the relatively rigid stents in the normally curved areas of the colon, ${ }^{6}$ or when the stents are place in eccentric positions. The ends of the stents can traumatize the normal colonic mucosa during peristalsis or in the case of flared colorectal stents, cause perforations from pressure necrosis at the ends of the stents. ${ }^{16}$ Perforation can also be related to stent migration with unresolved or recurrent bowel obstruction. ${ }^{23}$

Perforations are almost always managed by emergency surgical exploration $(65 \%)$. In some cases, limited perforations can be managed with bowel rest and broad spectrum antibiotics. Some limited perforations present as localized abscesses and percutaneous drainage combined with prolonged broad spectrum antibiotic therapy has been successful in most cases, avoiding a major surgery in these terminal patients (Fig. 9). As previously dis- cussed, a higher incidence of perforations is seen in patients receiving chemotherapy, especially in patients on Avastin. As the overall long-term survival of patients with colon cancer improves with newer chemotherapy regimens improves, the incidence of stent-related complications in likely to increase.

(3) Reobstruction: Colonic reobstruction is primarily reported in patients with colorectal stents placed for palliation. The median rate of reobstruction is $12 \%$ (range, 1-92\%). The majority of reobstructions result from tumor invasion. ${ }^{17}$ Rarely stent collapse or stent fracture can lead to reocclusions (Fig. 10). Stent obstructions occur from 48 hours to 480 days after placement and treatments included laser therapies to ablate obstructing tissue, restenting, surgery, and colonic irrigation. ${ }^{1,17}$ In most cases restenting provides a definitive solution. Covered stents have a lower incidence of tumor ingrowth but technical difficulties during deployment due to larger delivery systems, and a higher incidence of stent migration, have precluded a wider acceptance of this type of stents in the colon. Stoma creation may be ultimately required for patients that develop reobstruction when restenting and/or other minimally invasive alternative are not possible (Fig. 11). ${ }^{24}$

\section{REFERENCES}

1. Sebastian S, Johnston S, Geoghegan T, Torreggiani W, Buckley M. Pooled analysis of the efficacy and safety of self-expanding metal stenting in malignant colorectal obstruction. Am J Gastroenterol 2004;99:2051-2057.

2. Kim H, Kim SH, Choi SY, et al. Fluoroscopically guided placement of self-expandable metallic stents and stentgrafts in the treatment of acute malignant colorectal obstruction. J Vasc Interv Radiol 2008;19:1709-1716.

3. Baron TH. Interventional palliative strategies for malignant bowel obstruction. Curr Oncol Rep 2009;11:293-297.

4. Small AJ, Coelho-Prabhu N, Baron TH. Endoscopic place- 
ment of self-expandable metal stents for malignant colonic obstruction: long-term outcomes and complication factors. Gastrointest Endosc 2010;71:560-572.

5. Small AJ, Baron TH. Comparison of Wallstent and Ultraflex stents for palliation of malignant left-sided colon obstruction: a retrospective, case-matched analysis. Gastrointest Endosc 2008;67:478-488.

6. Song HY, Kim JH, Shin JH, et al. A dual-design expandable colorectal stent for malignant colorectal obstruction: results of a multicenter study. Endoscopy 2007; 39:448-454.

7. Lee KM, Shin SJ, Hwang JC, et al. Comparison of uncovered stent with covered stent for treatment of malignant colorectal obstruction. Gastrointest Endosc 2007;66: 931-936.

8. Caceres A, Zhou Q, Iasonos A, Gerdes H, Chi DS, Barakat RR. Colorectal stents for palliation of large-bowel obstructions in recurrent gynecologic cancer: an updated series. Gynecol Oncol 2008;108:482-485.

9. Keswani RN, Azar RR, Edmundowicz SA, et al. Stenting for malignant colonic obstruction: a comparison of efficacy and complications in colonic versus extracolonic malignancy. Gastrointest Endosc 2009;69:675-680.

10. Shin SJ, Kim TI, Kim BC, Lee YC, Song SY, Kim WH. Clinical application of self-expandable metallic stent for treatment of colorectal obstruction caused by extrinsic invasive tumors. Dis Colon Rectum 2008;51:578-583.

11. Jung MK, Park SY, Jeon SW, et al. Factors associated with the long-term outcome of a self-expandable colon stent used for palliation of malignant colorectal obstruction. Surg Endosc 2010;24:525-530.

12. Repici A, Adler DG, Gibbs CM, Malesci A, Preatoni P, Baron TH. Stenting of the proximal colon in patients with malignant large bowel obstruction: techniques and outcomes. Gastrointest Endosc 2007;66:940-944.

13. Stenhouse A, Page B, Rowan A, Giles L, Macdonald A. Self expanding wall stents in malignant colorectal cancer: is complete obstruction a contraindication to stent placement? Colorectal Dis 2009;11:854-858.

14. van Hooft JE, Fockens P, Marinelli AW, et al. Early closure of a multicenter randomized clinical trial of endoscopic stenting versus surgery for stage IV left-sided color- ectal cancer. Endoscopy 2008;40:184-191.

15. Fernandez-Esparrach G, Bordas JM, Giraldez MD, et al. Severe complications limit long-term clinical success of self-expanding metal stents in patients with obstructive colorectal cancer. Am J Gastroenterol 2010;105:1087-1093.

16. Song HY, Kim JH, Kim KR, et al. Malignant rectal obstruction within $5 \mathrm{~cm}$ of the anal verge: is there a role for expandable metallic stent placement? Gastrointest Endosc 2008;68:713-720.

17. Watt AM, Faragher IG, Griffin TT, Rieger NA, Maddern GJ. Self-expanding metallic stents for relieving malignant colorectal obstruction: a systematic review. Ann Surg 2007;246:24-30.

18. Khot UP, Lang AW, Murali K, Parker MC. Systematic review of the efficacy and safety of colorectal stents. $\mathrm{Br} \mathrm{J}$ Surg 2002;89:1096-1102.

19. Im JP, Kim SG, Kang HW, Kim JS, Jung HC, Song IS. Clinical outcomes and patency of self-expanding metal stents in patients with malignant colorectal obstruction: a prospective single center study. Int $\mathrm{J}$ Colorectal Dis 2008;23:789-794.

20. Wholey MH, Ferral H, Reyes R, Lopera J, CastanedaZuniga W, Maynar M. Retrieval of migrated colonic stents from the rectum. Cardiovasc Intervent Radiol 1997;20:477480.

21. Yoon CJ, Shin JH, Song HY, Lim JO, Yoon HK, Sung KB. Removal of retrievable esophageal and gastrointestinal stents: experience in 113 patients. AJR Am J Roentgenol 2004;183:1437-1444.

22. Garcia-Cano J, Sanchez-Manjavacas N, Gomez Ruiz CJ, et al. Endoscopic insertion of self-expanding metal stents in malignant colonic obstructions. Gastroenterol Hepatol 2006;29:610-615.

23. Kim JH, Song HY, Li YD, et al. Dual-design expandable colorectal stent for malignant colorectal obstruction: comparison of flared ends and bent ends. AJR Am J Roentgenol 2009;193:248-254.

24. Foo C, Poon JT, Law WL. Self-expanding metallic stents for acute left-sided large bowel obstruction: a review of 130 patients. Colorectal Dis. Forthcoming 2010. DOI: 10.1111/j.1463-1318.2010.02216.x. 